\title{
LAS CASAS DE ACOGIDA PARA MUJERES MALTRATADAS COMO RESPUESTA A UN PROBLEMA DE SALUD PÚBLICA ${ }^{1}$
}

ANA PAULA CID SANTOS

Universidad de Alicante

\section{INTRODUCCIÓN}

La O.M.S. establece diferentes pautas para la erradicación de la violencia de género, problema de salud pública que tan graves efectos tiene para el bienestar de tantas mujeres de todo el mundo. Una de sus recomendaciones aconseja elaborar estudios sobre la respuesta local a la violencia de género, y establecer así mecanismos más eficaces para su erradicación ${ }^{2}$.

En 1993, las Naciones Unidas definieron la violencia contra las mujeres como:

"Todo acto de violencia basado en el género que tiene como resultado posible o real un daño físico, sexual o psicológico, incluidas las amenazas, la coerción o la privación arbitraria de la libertad, ya sea que ocurra en la vida pública o en la privada ${ }^{3}$.

Desde entonces se ha venido dando un cambio fundamental en las diversas declaraciones internacionales que ofrecen, al respecto, un marco normativo para la actuación de los gobiernos del mundo, y que debe reflejarse en las políticas nacionales.

1. Este artículo es un resumen del trabajo "Casas de acogida en la provincia de Alicante. Contexto histórico e internacional». Premio Ayuda a la Investigación 2002, Instituto de Cultura Juan GilAlbert de la Diputación de Alicante, Premiado en su edición de 2004.

2. OMS: «Informe mundial sobre la violencia, 2002» (en www.who.int/mediacentre/events/2002/ es/index.html, consultado el 10 de febrero de 2004).

3. Conferencia Mundial de Derechos Humanos 1993. Naciones Unidas. Asamblea General, Junio de 1993, Austria (en www.ohchr.org/english/bodies/hrc/docs/ngos/cdr_shadow.pdf, consultado el 10 de febrero de 2004). 
La Conferencia Internacional sobre la Población y el Desarrollo ${ }^{4}$, la IV Conferencia Mundial sobre la Mujer ${ }^{5}$ y la Convención Interamericana para Prevenir, Sancionar y Erradicar la Violencia ${ }^{6}$ fueron ratificadas por la mayoría de los gobiernos.

La violencia basada en el género produce costos sanitarios, financieros y sociales, que en un país como Canadá se han calculado en 1.600 millones de dólares por año, en los que se incluye la atención médica y la pérdida de productividad. Según el Informe sobre el Desarrollo Mundial, publicado por el Banco Mundial en 1993, la violencia se sitúa entre las principales causas de la pérdida de años de vida entre mujeres ${ }^{7}$.

En España, el año 1997 marcó un punto de inflexión a partir del cual el problema de la violencia emerge en la agenda mediática con fuerza y obligó a actuaciones políticas más contundentes. Varios hechos contribuyeron a ello. Por una parte, el papel de las organizaciones de mujeres que venían denunciando las cifras de criminalidad. Por otra, el impacto que ocasionó la aparición en la Televisión andaluza de una mujer denunciando malos tratos, que días más tarde fue brutalmente asesinada por su marido, con quien compartía casa por orden judicial.

En este contexto, el I Plan de Acción contra la Violencia Doméstica se elaboró en 1998 y tuvo vigencia hasta el 2000, el II Plan Integral contra la Violencia Doméstica (2001-2004) fue aprobado en Consejo de Ministros del 11 de mayo de 2001, y la Ley Integral contra la Violencia de Género fue aprobada en diciembre de 2004.

Entre los recursos puestos en marcha para detener este problema de salud pública, se encuentran en un lugar destacado las denominadas casas de acogida para mujeres maltratadas, que son una respuesta a la necesidad de encontrar un refugio de urgencia por parte de muchas mujeres ante una situación de violencia. Las mujeres acuden a estos centros tras soportar años de maltrato, pierden su entorno social, y han de empezar una nueva vida, la mayoría de ellas con menores a su cargo.

\section{METODOLOGÍA Y TRABAJO DE CAMPO}

Esta investigación abarca todas las casas de acogida existentes en la provincia de Alicante. En el trabajo de campo, se han visitado todas estas casas de acogida y se ha entrevistado al personal directivo de cada centro, entre los días

4. Conferencia Internacional sobre la Población y el Desarrollo. Naciones Unidas. Desarrollo Económico y Social. Egipto, Septiembre de 1994.

5. Conferencia Mundial sobre la Mujer. Naciones Unidas. Documentos de Desarrollo Económico y Social. Beijing (China), Septiembre de 1995.

6. Conferencia Interamericana para Prevenir, Sancionar y Erradicar la Violencia. Belem (Brasil), Junio de 1994.

7. HeISE L.: "La Violencia contra la mujer en las Américas», Organización Panamericana de la Salud. Organización Mundial de la Salud. Subcomité de Planificación y Programación del Comité Ejecutivo, Octubre de 1998. 
23 de marzo y 5 de abril de 2004. En las entrevistas personales, se ha pasado un cuestionario con preguntas abiertas sobre el funcionamiento de las casas y su infraestructura.

Previamente al trabajo de campo, se llevó a cabo una revisión bibliográfica de los trabajos internacionales realizados y publicados sobre las casas de acogida, así como de los trabajos de ámbito nacional publicados en España. La búsqueda se realizó en bases de datos internacionales, utilizando las palabras claves: shelter, battered, women.

Dado que en la búsqueda no aparecieron artículos con referencia a la situación de las casas de acogida en España, para identificar las investigaciones llevadas a cabo sobre la situación en el país se utilizó el buscador Google y las palabras clave: casas de acogida y mujeres maltratadas. Además, se contó con la información extraída de la Hemeroteca del Instituto de la Mujer. Se obtuvo información sobre tres estudios de ámbito nacional sobre las casas de acogida para mujeres maltratadas: el último data de 1996, como veremos más adelante. No se ha encontrado ninguna publicación que trate específicamente sobre las casas de acogida de la provincia de Alicante.

\section{3. ¿CÓMO SURGEN LAS CASAS DE ACOGIDA PARA MUJERES MALTRATADAS?}

La lucha para erradicar la violencia contra las mujeres comienza en la década de los setenta. En estos años, en los Estados Unidos cada 30 segundos una mujer era golpeada por su compañero, cónyuge o ex-marido, y un tercio de las mujeres víctimas de homicidios eran asesinadas por sus propios maridos o amantes. En el $80 \%$ de los casos la policía había sido llamada al menos una vez, para intervenir en las situaciones de violencia doméstica antes de que se desencadenara el crimen. El maltrato a las mujeres y los abusos a menores se consideraban ya entonces el mayor problema social, y los estudios se centraban en interpretar el significado social de la violencia en la familia, sus causas y las estrategias para combatirla ${ }^{8}$.

El movimiento de casas de acogida no sólo fue una primera respuesta a la violencia doméstica. Su objetivo estratégico consistía en cuestionar el modelo familiar vigente y se planteaba como tarea definir las formas familiares donde no existía violencia y donde se había completado la ruptura con un modelo patriarcal ${ }^{9}$.

Los objetivos comunes compartidos por las casas de acogida creadas en ese momento eran: promover un ambiente de cuidados y seguridad para las mujeres maltratadas y sus hijos; ofrecerles soporte emocional y asesoramiento socio psicológico; proveer información sobre los derechos legales de las mujeres; dar

8. MCGRATH, C.: "The crisis of domestic order», Socialist Review, 43 (1979), pp. 11-30.

9. EPSTEIN, S.: "Structure and ideology of shelters for battered women", American Journal of Comunity. Psychology, 16 (1998), pp. 345-367. 
asistencia en las comparecencias ante los juzgados; aportar opciones de vivienda, y explorar objetivos y direcciones a tomar en una vida futura ${ }^{10}$.

El movimiento de casas de acogida para mujeres maltratadas que surge en estos años estuvo muy influido por los sectores feministas que tomaron la iniciativa y la organización de muchos de estos centros. Cuando se planteó el desarrollo de estos servicios, el objetivo no era sólo ayudar a las mujeres, sino construir espacios y organizaciones que mantuvieran los valores de participación e igualdad en todas las personas. Muchas casas de acogida adoptaron una estructura colectivista y no jerárquica. Las normas fueron minimizadas y las decisiones eran tomadas por consenso. Las residentes participaban activamente en el desarrollo de los programas que se llevaban a cabo. Dado que se potenciaba la auto-ayuda, antiguas residentes eran animadas a volver como trabajadoras para dar soporte y servir como modelos de mujer que habían conseguido tener el control sobre sus vidas ${ }^{11}$. Estas casas encontraron pronto problemas para su financiación y la lentitud del proceso de consenso les llevó a contradicciones entre los valores a mantener y la práctica del día a día. No sólo ofrecían refugio a las víctimas de la violencia sino que llamaron la atención pública sobre la difícil situación que estas personas tenían que soportar ${ }^{12}$.

La razón por la que la violencia doméstica emerge en este momento histórico concreto -los años setenta-como problema social se hallaría en el hecho de la mayor presencia de las mujeres en el mercado laboral, y de su mayor poder en las esferas legal y cultural. A esto se añadiría el declive de la institución familiar como instrumento de dominación femenina. También se une a ello la fuerza del movimiento feminista, que eligió atacar el punto débil, y no el fuerte, de la dominación masculina ${ }^{13}$.

En 1982 se habían creado en los Estados Unidos 300 centros de acogida y se había reconocido el "síndrome de la mujer maltratada» dentro de la Clasificación Internacional de Enfermedades ${ }^{14}$. El movimiento de casas de acogida para mujeres maltratadas representó uno de los que más rápidamente se expandió a través de toda la nación, desde la movilización contra la guerra de Vietnam ${ }^{15}$. A medida que la sociedad fue tomando conciencia del problema, las agencias federales, estatales y locales del estado de bienestar empezaron a hacerse cargo y financiar estos centros, lo que no dejaba de ser un triunfo, ya que daba la posibilidad de acceder a mayores fuentes de financiación hacía crecer la visibilidad de las mujeres maltratadas ante la sociedad. Pero también se pagó un precio, pues la financiación estatal supuso abandonar el tipo de organización

10. ROBERTS, A.: «Sheltering battered women: A national study and service guide», The Social Science, 3(1981), pp. 88-100.

11. MORGAN, P.: "From battered wife to program client: The state shaping of social problems», Kapitalistate, 9 (1981), pp. 17-39.

12. SCHILLINGER, E.: "Dependency, control and isolation. Battered women and the welfare system", Journal of Contemporary Ethnography, 16 (1988), pp. 469-490.

13. EASTON, B.: «Feminism and the contemporary family», Socialist Review, 39 (1978), pp. 67-79.

14. SCHILLINGER, E.: Op. cit.

15. MORGAN, P.: Op. cit. 
Las casas de acogida para mujeres maltratadas como respuesta a un problema de...

colectivista y de democracia directa en aras de una mayor profesionalización y burocratización ${ }^{16}$.

\section{LA SITUACIÓN EN ESPAÑA}

En España, la génesis de estas instituciones es diferente a la de otros países desarrollados, pues comienzan a crearse en 1984, y parten de la experiencia de los modelos que ya estaban en funcionamiento en Europa. Las casas de acogida en España se crean directamente por los Ayuntamientos y las comunidades autónomas ${ }^{17}$. En más de veinte años de experiencia se dispone de poca documentación sobre su funcionamiento, a pesar de que, en la actualidad, existen más de 125 centros de acogida en toda España.

La inversión anual en centros es de 1,8 millones de euros. La mayor parte de esta financiación proviene del impuesto sobre la renta ${ }^{18}$. Hay una gran variación en la financiación de estos programas de acogida, algunos cuestan 30.000 euros, otros se financian con 126.000 euros $^{19}$. Las cifras que se manejan en ocasiones engloban tanto a los centros de acogida como a los albergues, los pisos tutelados y pisos de emergencia que, en principio, tienen diferentes objetivos ${ }^{20}$. La Comisión del Parlamento Europeo para los Derechos de las Mujeres recomendaba en 1987 una plaza por cada 10.000 habitantes. En España hay una por cada 310.000 habitantes, según datos de 1998.

El primer trabajo que se publica en España data de 1982 y es anterior a la puesta en marcha de este tipo de centros ${ }^{21}$. En ese momento, se recogían 1.300 denuncias mensuales de malos tratos. Las mujeres tras la denuncia volvían al hogar familiar; no existían soluciones de emergencia. Este informe se elaboró tras la visita a diferentes centros en Inglaterra y Francia. En el Reino Unido existía ya entonces una Federación Nacional de refugios para mujeres maltratadas que agrupaba a 200 casas entre Inglaterra, Escocia y País de Gales. La finalidad de este informe realizado por el Instituto de la Mujer era que sirviera como documentación básica para aquellas instituciones y organismos españoles que estuvieran interesados en la creación de casas de acogida para mujeres maltratadas. Seis años más tarde, en 1992, el Instituto de la Mujer publica un informe en el que se recoge la experiencia de 25 casas que ya están trabajando en distintas poblaciones españolas ${ }^{22}$. Es un amplio estudio con una metodolo-

16. FERRARO K-J.: «Negotiating trouble in a battered women's shelter», Urban-Life, 5 (1983), pp. 345-360.

17. WAlkeR, P.; ReY, C.; Segovia, J.; Alonso, A.: Informe sobre casas de acogida para mujeres que sufren malos tratos, Ministerio de Cultura-Instituto de la Mujer, Madrid, 1986.

18. Diario de sesiones de las Cortes Generales. De los derechos de la mujer, no 46, 2001.

19. De La Fuente, C; Montraveta, I.; Valls, R.: Quién es quién. Las entidades de acción social beneficiarias de la asignación tributaria del 0,52\% del IRPF, Ministerio de Trabajo y Asuntos Sociales. Subdirección General de Publicaciones, Madrid, 1998.

20. ARRANZ F.: Las políticas públicas a favor de las mujeres, Instituto de Investigaciones Feministas de la Universidad Complutense de Madrid, Madrid, 2000.

21. Walker, P.; Rey, C.; Segovia, J.; Alonso, A.: Op.cit.

22. Ibídem. 
gía cuanti-cualitativa. En él, se realiza un análisis del perfil socioeconómico, tanto de la mujer maltratada como del maltratador y del tipo de maltrato que llega hasta estos centros. Se recoge, así mismo, datos sobre la infraestructura, localización, funcionamiento y equipo de trabajo de los centros. Se analizan los puntos críticos y los fuertes que en ese momento se encuentran, y las autoras proponen al final del mismo un nuevo modelo de referencia, basándose en los datos obtenidos. En este trabajo también se confrontaron experiencias con casas de acogida situadas en Estocolmo, Londres y Bruselas.

El último trabajo de ámbito nacional que se ha encontrado corresponde a $1996^{23}$. Recoge información a través de cuestionarios de cincuenta casas de acogida en todo el territorio español. Este informe hace especial hincapié en la heterogeneidad que existe entre las casas de acogida en lo referente a la gestión, capacidad, medios económicos, equipos de profesionales, recursos y criterios de admisión. Mantiene que no existe una situación análoga en todas las Comunidades Autónomas y aboga por unificar criterios.

De este trabajo se extraen algunos datos de especial relevancia. Son los siguientes: Sólo el $46 \%$ de la muestra es un servicio exclusivo para mujeres maltratadas, el $54 \%$ restante acoge a mujeres con diferentes problemas. Un $28 \%$ se gestiona por los ayuntamientos, el $26 \%$ por Comunidades Religiosas, el $14 \%$ por Asociaciones de mujeres, el $4 \%$ por la Confederación de Asociaciones de Vecinos y el $28 \%$ restante es gestionado por diferentes instituciones. El 56\% no desarrolla ninguna actividad específica con las mujeres y los niños; un $32 \%$ realiza talleres de Cerámica, Corte y Confección; un $12 \%$ organiza terapias grupales o individuales. En este trabajo se plantea que el $60 \%$ de las mujeres que residen en una casa de acogida vuelve con el maltratador.

Desde ese año de 1996 no se tiene constancia de ningún otro trabajo de ámbito nacional que evalúe la situación en la que se encuentran las casas de acogida.

Las casas de acogida son sólo una medida provisional que debe venir acompañada de otras medidas de reinserción, pues lo que aparentemente es una medida de protección, implica en realidad posicionar a las mujeres en una situación de revictimización y, en definitiva, supone una restricción de los derechos de la víctima, no de los agresores, y son éstos los que deben sufrir las consecuencias y asumir las responsabilidades de sus actos, y por lo tanto, han de ser sujetos de las órdenes de expulsión de los domicilios familiares ${ }^{24}$.

23. "Casas de acogida para mujeres maltratadas», Comisión para la Investigación de Malos Tratos a las Mujeres. Instituto de la Mujer, Madrid, 1996.

24. Delgado Cordero, Ana: "Alternativas para combatir la violencia doméstica», Ponencia en la Mesa redonda sobre violencia de género, presentada en el IV Congreso de Escuelas de Trabajo Social, Alicante, abril 2002 (en Alternativas. Cuadernos de trabajo social, 10 (2002), pp. 167-173). 


\section{LA SITUACIÓN DE LAS CASAS DE ACOGIDA EN LA PROVINCIA DE ALICANTE}

Atendiendo a la tipología establecida en la orden del 17 de febrero de 2003, en la provincia de Alicante existen:

- Una casa de acogida con carácter de emergencia.

- Seis centros de acogida.

En la siguiente relación se denomina a cada casa por la organización que la gestiona, y se reseñan algunas de sus principales características:

\section{Emaus}

Organización fundada por el sacerdote católico francés Abbé Pierre, miembro de la resistencia durante la segunda guerra mundial y diputado entre 19471951. En la actualidad es una asociación estatal laica.

Centro de acogida: Casa con amplio jardín situado a las afueras de un núcleo urbano de 16.000 habitantes, en la Marina Baixa.

Capacidad máxima para 8 mujeres y 9 niños.

\section{Adoratrices Esclavas de Santísimo Sacramento y de la Caridad.}

Orden religiosa constituida el 1 de enero de 1857. Es una organización de ámbito estatal que trabaja con colectivos de mujeres, drogodependientes, personas reclusas y ex reclusas. El centro que gestiona en Alicante está en funcionamiento desde 1988.

Centro de acogida: Dos pisos unidos dentro de un complejo religioso, en pleno casco urbano.

Capacidad máxima para 9 mujeres y 11 niños, de 0 a 7 años.

\section{Mensajeros de la Paz}

Fundada en 1972 por el sacerdote Ángel García Rodríguez, es «una organización de carácter laico progresista», según el Directorio ONGD 2000, de la coordinadora de ONGs de España.

Gestiona la casa de emergencia constituida por dos pisos unidos. Se encuentra situada dentro de un gran bloque de viviendas, en un barrio de la periferia.

Capacidad para 6 mujeres y 6 niños. Admiten adolescentes, pero en el caso de los varones se valora su posible admisión en la casa.

\section{Mujeres por la Democracia}

Federación de ámbito estatal, dirección Calle Génova, 13, creada el 10 de agosto de 1992. Atiende a los siguientes colectivos: jóvenes, mujeres y personas con minusvalías.

Gestiona una casa de acogida formada por dos pisos unidos dentro de un bloque pequeño de viviendas. Situado en el núcleo urbano de una población de 50.000 habitantes en la Vega Baja.

Capacidad para 4 mujeres y 8 niños. Los niños son admitidos hasta los 14 años. 


\section{Gesmed (Gestión Socio-sanitaria del Mediterráneo)}

Según informa en su página web,

"Gesmed surge con el objetivo de desarrollar la gestión socio-sanitaria del Grupo Imedes como respuesta a las nuevas necesidades generadas en un mercado de rápido crecimiento: los servicios de atención a personas. Ayuda a domicilio, residencias de personas mayores, discapacitados, menores, mujeres maltratadas y otros programas de acción comunitaria dirigidos a colectivos con necesidades específicas ${ }^{25}$.

Tiene un convenio hasta el año 2006.

Gestiona un centro de acogida situado en un centro urbano.

Capacidad para 9 mujeres y 18 menores. En principio no hay límite de edad para los menores, pero se valora en cada caso. El límite está en los 14 años para los varones, pero se ha saltado esta condición con frecuencia.

\section{Fundación Nuestra Señora del Carmen}

Es un Patronato, cuyo presidente es el alcalde de la localidad donde está ubicada, que posee 6.000 habitantes y está situada en el interior de la comarca de la Marina Alta.

Casa de acogida: Edificio antiguo anexo a una residencia de ancianos.

Capacidad máxima de 7 plazas para niños y 7 para mujeres. Por la proximidad con la residencia no se admiten niños mayores de 8 años.

\section{Hermanas Oblatas del Santísimo Redentor}

En 1870 se convierte en congregación religiosa. Desde entonces interviene en barrios periféricos, zonas donde se ejerce la prostitución, atención a adolescentes y jóvenes en situación de riesgo, madres solteras y mujeres maltratadas.

Casa de acogida: Dos pisos unidos dentro de un complejo religioso, situado en un núcleo urbano.

Capacidad para 13 mujeres (seis en un piso y siete en otro). Se admiten menores de 18 meses.

\section{Total de plazas en la provincia de Alicante}

Para mujeres: 56.

Para niños: 66.

Total de ingresos anuales: 158 mujeres y 202 menores.

\section{CONCLUSIONES}

Tras analizar las respuestas obtenidas de las diferentes entrevistas, este estudio llega a las siguientes conclusiones:

- Las últimas leyes y órdenes establecidas por la Generalitat Valenciana con fecha de 2003 unifican criterios en cuanto a la organización y funcionamiento de estos centros.

25. http://www.gesmed.es (consultado el 20 de marzo de 2007). 
- El $83 \%$ de estas mujeres y sus hijos llegan a las casas a través de los Centros 24 Horas y de los Servicios Sociales municipales.

- Las organizaciones y el personal directivo que gestiona estos centros mantienen diferentes criterios en cuanto a las causas y soluciones de la violencia de género.

- No existe en la provincia de Alicante un centro exclusivo para mujeres maltratadas que atienda a estas mujeres de manera integral. Tampoco hay viviendas tuteladas, que es una de las tipologías estipuladas por la orden de 17 de febrero de 2003 de la Consellería de Bienestar Social de la Comunidad Valenciana.

- El 30\% de los ingresos en estos centros son mujeres, con o sin cargas familiares, que carecen de recursos, y el motivo de su ingreso no es la violencia. Por malos tratos ingresa el $51,21 \%$, por prostitución el $6 \%$ y un $12 \%$ entra por el apartado de otros motivos.

- Solo una minoría de las mujeres que sufren violencia acceden a las casas de acogida.

- El perfil socio-económico y educativo de las mujeres que ingresan en estos centros es bajo. Casi el $44 \%$ no ha obtenido el graduado escolar.

- Las mujeres inmigrantes, por su mayor precariedad socioeconómica, ocupan en el momento del estudio casi el $50 \%$ de las plazas en estos centros.

- El déficit de guarderías, el difícil acceso a una vivienda y la precariedad laboral son los problemas que se destacan como más importantes; son recursos que siguen sin estar al alcance de estas mujeres, a pesar de que en los trabajos y estudios de los años ochenta ya se alertaba sobre la cuestión.

- La carencia de estos medios externos a las casas (guarderías, vivienda, trabajo...) disminuye la eficacia de este recurso.

- Las casas de acogida se encuentran, por lo general, insuficientemente financiadas, lo que repercute también en su funcionamiento.

- Se desconoce el número de mujeres que consiguen rehacer sus vidas de una forma autónoma, después de pasar por los programas de intervención de una casa de acogida. 